\title{
Alter
}

Revue de phénoménologie

$18 \mid 2010$

L'Attention

\section{Attention et pertinence chez Alfred Schütz}

\section{Laurent Perreau}

\section{OpenEdition}

Journals

Édition électronique

URL : http://journals.openedition.org/alter/1572

DOI : $10.4000 /$ alter.1572

ISSN : 2558-7927

\section{Éditeur :}

Association ALTER, Archives Husserl (CNRS-UMR 8547)

\section{Édition imprimée}

Date de publication : 1 octobre 2010

Pagination : 79-92

ISBN : 2-9522374-6-8

ISSN : $1249-8947$

Référence électronique

Laurent Perreau, "Attention et pertinence chez Alfred Schütz », Alter [En ligne], 18 | 2010, mis en ligne le 01 juin 2020, consulté le 29 juin 2020. URL : http://journals.openedition.org/alter/1572 ; DOI https://doi.org/10.4000/alter.1572 


\title{
ATTENTION ET PERTINENCE CHEZ ALFRED SCHÜTZ
}

\author{
Laurent Perreau
}

La philosophie a souvent pensé l'attention en référant celle-ci à la volonté et à la perception. Elle décline ainsi deux paradigmes fondamentaux qui abordent la question de l'attention à partir de présupposés qui, pour décisifs qu'ils soient, ne sont pas toujours discutés pour eux-mêmes.

Le paradigme volontariste fait de l'attention le produit d'un acte de la volonté, fût-ce sous une forme émergente. De Descartes à Alain, en passant par Maine de Biran et Bergson, l'attention est ainsi définie comme cet effort mis par la conscience dans la considération de ses divers objets : l'attention n'est rien si on ne la fait, ni ne la veut, d'une manière ou d'une autre. L'inauguration du champ de conscience procède d'une application particulière de la volonté qui s'impose aux forces obscures de l'inattention, de l'inconscient et du non-conscient ${ }^{1}$.

À ce premier paradigme s'en ajoute un second, qui pense l'attention comme une activité de l'esprit spécifiquement liée à celle de la perception consciente. Selon ce paradigme, qui l'on peut dire par commodité intentionnaliste, l'attention est cette faculté qui assure la focalisation de la conscience sur l'objet pris en considération. L'attention n'est donc pas seulement la condition d'un rapport éveillé au monde. Elle opère, au sein du champ de conscience, une forme de mise en relief qui délimite une aire de focalisation thématique. En outre, son exercice maintenu conditionne l'inspection des singularités de l'objet. L'attention est donc cette fonction

1. Voir par exemple, chez Descartes, les Règles pour la direction de l'esprit, III et IX, les Principes de la philosophie, I, 73 ; chez Bergson, Essai sur les données immédiates de la conscience, Paris, PUF, 2007, p. 20 sq., où l'attention est définie comme « effort intellectuel». Voir aussi D. Kahneman, Attention and Effort, Prentice-Hall, Englewood Cliffs (New Jersey), 1973. 


\section{L'attention}

cognitive qui garantit la pleine présence de l'objet à la conscience. C'est à ce titre qu'elle joue un rôle moteur dans l'expérience perceptive ${ }^{2}$.

Mais en abordant de cette manière l'attention par différence, en la spécifiant progressivement par rapport à la volonté et à la perception, ne risque-t-on pas de manquer certaines de ses caractéristiques positives ? On est notamment en droit de se demander si ce double paradigme ne confine pas d'emblée la considération philosophique de l'attention à une appréhension trop résolument individualiste : en pensant l'attention par le biais de ce qu'il peut y avoir de volontaire en elle ou encore par le rapport qu'elle entretient avec la perception, ne sommes-nous pas condamnés à ne voir dans l'attention qu'un acte procédant du sujet individuel, dans la solitude de son rapport au monde? Ne convient-il pas de thématiser également les conditions sociales de l'exercice de l'attention, en articulant plus finement la théorie de l'attention à une théorie de l'expérience sociale du sujet? Par ailleurs, on peut s'interroger sur l'insistance mise à faire de l'attention un acte ou encore le produit d'une activité. Cet abord de l'attention ne nous invite guère à reconsidérer la part de passivité avec laquelle l'attention compose nécessairement: n'y aurait-il pas là quelque ressource pour resituer l'attention dans le cours de la vie consciente, en montrant mieux ce qui structure et conditionne son fonctionnement dans l'ordre de la passivité ?

À cet égard, les réflexions qu'Alfred Schütz a pu consacrer à la question de l'attention présentent au moins deux intérêts. D'une part, Schütz développe une théorie de l'attention qui emprunte à la phénoménologie comme au pragmatisme, dans une sorte d'entre-deux théorique qui présente l'insigne avantage de ne pas subordonner immédiatement la question de l'attention à celle de la perception : selon lui, l'attention est d'abord et avant tout une activité de la conscience en prise directe avec notre inscription pratique dans le monde. D'autre part, Schütz évite une conception outrancièrement individualiste de l'attention en pensant celle-ci sous la rubrique novatrice de la pertinence : l'attention demeure un acte du sujet, mais elle se trouve repensée à partir des "systèmes de pertinence ", où se révèlent les structures et les conditions socialement partagées sous lesquelles s'exercent l'attention. L'attention n'est alors plus seulement pensée dans l'horizon de la subjectivité individuelle et de l'intersubjectivité (une perspective dont les discussions récentes relatives au phénomène de la joint attention ont montré toute la fécondité3) mais plus proprement dans sa dimension sociale.

Dans le cadre du présent article, nous restituerons tout d'abord l'analyse phénoménologique de l'attention développée par Schütz dans sa thèse de

2. Ce paradigme trouve sa déclinaison phénoménologique dans le $\S 92$ des Idées directrices I de Husserl (tr. P. Ricœur, Paris, Gallimard, 1985) ou encore dans le Husserliana XXXVIII. Wahrnehmung und Aufmerksamkeit, éd. par T. Vongehr, R. Giuliani, Dordrecht, Kluwer, 2004.

3. Nous renvoyons au bel article d'E. Bimbenet présenté dans ce même numéro. 
1932 pour montrer ensuite comment cette théorie de l'attention a trouvé un prolongement singulier dans sa théorie des structures de pertinence.

\section{L'esquisse d'une phénoménologie de l'attention}

Les premières réflexions que Schütz consacre à la question de l'attention se trouvent au paragraphe 13 de sa thèse parue en 1932 et intitulée Der sinnhafte Aufbau der sozialen Welt. Eine Einleitung in die verstehende Soziologie 4 . Le projet de Schütz est alors celui d'une fondation philosophique des sciences sociales et plus précisément de la méthodologie de la sociologie développée par M. Weber dans Économie et sociétét

Aux yeux de Schütz, le premier mérite de M. Weber tient à ce qu'il nous évite les coûteuses hypostases de $l^{\prime}$ « esprit objectif » en revenant au comportement individuel et au "sens" subjectivement visé par l'agent. Weber parvient ainsi à définir le domaine d'objets de la sociologie en considérant l'activité de compréhension individuelle et interindividuelle comme une donnée première qui constitue le matériau propre de l'analyse sociologique. Le second mérite des vues de M. Weber concerne la méthodologie des sciences sociales. En effet, la scientificité de la sociologie compréhensive ne peut être validée que dans la référence maintenue à cette donnée première de la compréhension subjective et dans sa réélaboration conjointe aux moyens de procédures rigoureuses qui vont en conduire l'explicitation et l'explication. Ainsi, Weber montre en quoi la possible objectivité de la sociologie dite « compréhensive » dépend de procédures qui peuvent être méthodologiquement définies.

Cependant, s'il approuve les résultats de l'approche wébérienne, Schütz entend les confirmer et les conforter en reprenant l'élaboration conceptuelle de ses concepts fondamentaux : l'«agir social» (soziales Handeln, l'agir

4. A. Schütz, Der sinnhafte Aufbau der sozialen Welt. Eine Einleitung in die verstehende Soziologie (1929-1932), Vienne, Springer, 1932, 2e éd., 1960. Nous citons systématiquement la deuxième édition du texte en utilisant l'abréviation Aufbau. Il existe une édition au format de poche: Alfred Schütz, Der sinnhafte Aufbau der sozialen Welt, 6e édition, Francfort, Suhrkamp, 1993. Il existe également une traduction en langue anglaise: The Phenomenology of the Social World, trad. par G. Walsh et F. Lehnert, avec une introduction de G. Walsh, Evanston (Illinois), Northern University Press, 1967. Nous citons ci-après les textes suivants : A. Schütz, Collected Papers I. The Problem of Social Reality, The Hague, Martinus Nijhoff, 1962 (abrégé CPI) ; Collected Papers II. Studies in Social Theory, The Hague, Martinus Nijhoff, 1964 (abrégé CPII) ; Collected Papers III. Studies in Phenomenological Philosophy, The Hague, Martinus Nijhoff, 1966 (abrégé CPIII); Collected Papers IV, Den Haag, Kluwer Academic Publishers, 1996 (abrégé CP IV). Certains de ces textes ont été traduits en français et rassemblés en deux recueils : A. Schütz, Le chercheur et le quotidien, Paris, Klincksieck, 1987 (abrégé CQ) et Éléments de sociologie phénoménologique, Paris, L'Harmattan, 1998 (abrégé ESP). Pour une introduction très complète à l'œuvre de Schütz, cf. D. Cefaï, Phénoménologie et sciences sociales. Alfred Schütz. Naissance d'une anthropologie philosophique, Genève, Librairie Droz, 1998.

5. M. Weber, Économie et société. Tome I : les catégories de la sociologie, Paris, Pocket, 2003. 


\section{L'attention}

orienté en fonction du comportement des autres membres du monde social), le «sens » subjectivement visé (le sens considéré in statu nascendi, comme produit d'une conscience individuelle), etc. Cette entreprise s'autorise d'une analyse constitutive de l'expérience subjective qui procède elle-même d'une phénoménologie de l'attitude naturelle ${ }^{6}$. C'est dans ce cadre bien particulier que Schütz élabore une phénoménologie de l'attention qui diffère notablement de celle développée par Husserl.

Schütz conçoit la conscience comme "courant de l'expérience vivante », comme un flux ininterrompu de vécus (Erlebnisse), définition où s'atteste, sous la référence à Husserl, une certaine fidélité à Bergson. En effet, Schütz retient de Bergson une conception de la conscience qui la comprend comme temporalité ou durée. De manière tout à fait frappante, la définition de la conscience ne s'ordonne pas à la conception phénoménologique classique, celle de la conscience intentionnelle comme conscience de..., comme foyer des actes objectivants. Pour Schütz, la conscience est avant tout vie et durée, elle est un flux d'expériences vécues hétérogène qui ne cesse de s'écouler, en présentant une multiplicité concrète d'états qui sont intensivement vécus. Pure continuité, écoulement incessant, la conscience est donc indivisible, indéfinie.

Toute la question est alors celle de l'accès à la phénoménalité et partant, celle de la constitution du sens de l'expérience. Selon Schütz, dans l'expérience pré-phénoménale et pré-immanente, les vécus ne sont pas distingués les uns des autres : aucun «sens » ne peut leur être attribué et encore moins faire l'objet d'une quelconque compréhension. La détermination du sens va donc dépendre ici entièrement du repérage de l'identité discrète d'un vécu qui le détache $d u$ " courant du conscience ${ }^{7}$ ». Pour rendre compte des procédures d'identification des vécus au sein du flux de la conscience, Schütz mobilise alors une théorie de la réflexion attentionnelle qui se rapporte à la durée en y instruisant des partitions. La durée intime de la conscience est par principe irréfléchie: $l^{\prime}$ intervention réflexive de l'attention consiste précisément à interrompre le cours de l'expérience, du vivre, pour lui conférer un sens et en faire une expérience vécue.

En effet, que disons-nous précisément lorsque nous disons qu'une expérience fait sens ou qu'elle a du sens? Dire cela, ce n'est pas constater que l'expérience possède un sens, car le sens de l'expérience n'est pas une propriété objective. À l'évidence, il ne suffit pas non plus qu'une expérience soit vécue pour qu'elle fasse sens. Selon Schütz, elle fait sens à partir du

6. Sur cet aspect particulier de l'œuvre de Schütz, cf. Bruce Bégout, « Alfred Schütz et l'épochè de l'attitude naturelle », Alter, 11, 2003, p. 165-192. Schütz conçoit la phénoménologie comme une psychologie intentionnelle apte à décrire les structures eidétiques qui régissent les phénomènes de sens «dans la socialité mondaine ». Elle se développe sous la forme d'une analyse réflexive de l'attitude naturelle. Cf. : Aufbau, p. 41-42.

7. «Les vécus du monde matériel et du monde interne au sein desquels nous vivons sont en soi d'ordre pré-phénoménal et [...] ils ne sont phénoménalisés que par un acte particulier de la conversion attentionnelle (Zuwendung) ». Aufbau, § 14, p. 79. 
moment où elle se détache du flux de la conscience, comme mise en relief par un acte de l'attention. C'est cette mise en exergue qui en fait un point particulier autour duquel se redéfinit le courant de conscience. Le sens, à ce point de l'analyse, se définit donc comme une structure d'orientation singularisée de la vie subjective 8 .

Dans le cadre de cette définition, le rôle de l'attention est celui d'une sélection qui opère par rapport à la multiplicité des données et des expériences de la conscience. L'acte d'attention peut dès lors être assimilé à un "cône de lumière " projeté sur le flux de la conscience, faisant ressortir les contours et les détails d'une expérience particulière du flux de conscience ${ }^{9}$. En d'autres termes, l'attention est ce «rayon du regard » qui s'applique à tel endroit ou moment du flux pour le porter à pleine lumière et rejeter dans l'ombre ce qui ne retiendra pas l'attention ${ }^{10}$.

On constate donc à ce point que l'attention - tel est bien l'intérêt de son investigation phénoménologique - n'est pas prioritairement pensée par rapport au thème, mais bien par rapport aux vécus auxquels elle s'applique. L'attention n'est pas une faculté de l'esprit, un pouvoir de connaître : elle est un acte qui distingue telle expérience plutôt que telle autre sur le fond du flux de la conscience temporelle.

L'attention, ainsi conçue, présente plusieurs caractéristiques phénoménologiques dont le repérage esquisse une théorie cohérente :

1. L'attention est un acte qui engendre une modification des vécus de conscience. Schütz retient à cet égard les remarques formulées par Husserl à propos des modifications attentionnelles dans le livre I des Idées directrices ${ }^{11}$, où Husserl insiste sur le fait que l'attention suscite des modifications d'ordre noématique et noétique. Schütz reprend cette idée dans la perspective d'une refondation philosophique de la méthodologie weberienne: $c^{\prime}$ est à travers une «conversion attentionnelle» (Zuwendung) que certains vécus sont distingués, portés au regard pour sceller le sens de telle action ou de tel projet ${ }^{12}$.

2. L'attention est un acte essentiellement rétrospectif. Il n'y a pas d'attention spontanée, immédiatement corrélée au présent vivant et à

8. «Le sens est avant tout la caractérisation d'une orientation déterminée du regard portant sur une expérience vécue en propre, dans le se-laisser-vivre pur et simple du déroulement de la durée, vécu que nous pouvons « détacher » de tous nos autres vécus seulement au moyen d'un acte réflexif, jusqu'à le délimiter entièrement. Le sens caractérise ainsi une attitude particulière du moi à l'égard du déroulement de sa durée. "Aufbau, §6, p. 40. Cette définition du sens est ensuite complétée par Schütz pour satisfaire aux réquisits de la méthodologie wébérienne. Notons que cette théorie du sens diffère de celle de Husserl pour qui la détermination du sens ne dépend pas d'une théorie de la réflexion attentionnelle, mais d'une théorie de l'intentionnalité. 9. Cette métaphore n'est pas originale, elle est fréquente dans la littérature psychologique de l'époque.

10. Aufbau, § 12 , p. 73 .

11. E. Husserl, Idées directrices pour une phénoménologie et une philosophie phénoménologique pures. I, Paris, Gallimard, 1950, § 92, p. 317-318.

12. Aufbau, § 12, p. 74. 


\section{L'attention}

l'épaisseur du vécu. L'attention ne porte pas sur le présent vivant, qui est ineffable. Elle ressaisit après coup, phase par phase, les différents moments de l'expérience. L'attention est un acte qui récapitule le cours de $l^{\prime}$ expérience ${ }^{13}$.

2. L'attention est un acte réflexif: elle réfléchit à sa manière l'impression originaire du présent vivant en opérant sur elle une remémoration active et reproductive, en la réactivant et en la reconfigurant. En ce sens, l'attention qui scelle le sens de l'expérience vécue est distincte de la rétention, cette "queue de comète» du présent vivant qui est encore, à ce titre, partie intégrante du présent vivant ${ }^{14}$. Pour autant, cette réflexivité ne la réduit pas à un simple reflet qui viendrait dupliquer l'expérience.

3. L'attention est discontinuiste: elle opère toujours sur le mode de la coupure. Tandis que le jeu continu des rétentions et des protentions préserve sans interruption le courant du flux de conscience, l'attention instaure une solution de continuité. La conversion attentionnelle (Zuwendung) a pour fonction de distinguer le vécu et, du même coup, son sens subjectivement visé. L'héritage des conceptions bergsoniennes est patent: l'application réflexive de l'attention opère des "coupes» au sein du courant de conscience et scelle l'identification des différents vécus. L'attention accordée à telle expérience décide donc d'un Maintenant, d'un Ici et d'un Ainsi, c'està-dire d'une ponctualité temporelle, d'une définition spatiale et d'une détermination particulière des modalités du rapport à l'expérience ${ }^{15}$.

4. L'attention est un acte objectivant qui met en relief des objets d'expérience en leur conférant une certaine unité. L'objet de l'attention est ultimement le thème expérientiel mis en relief et la thématisation de l'objet sanctionne la réussite de l'attention. Mais avant cela, il faut aussi reconnaître que l'attention découpe des unités discrètes qui donnent accès au phénoménal et qui portent en germe la définition du sens de l'expérience. Il y a en ce sens des «synthèses polythétiques » qui précèdent l'établissement des «synthèses monothétiques » : les synthèses polythétiques délimitent un champ phénoménal, tandis que les synthèses monothétiques les unifient et constituent ainsi des objets d'expérience qui pourront être visés de diverses manières. Le thème, l'objet visé, manipulé, utilisé (etc.), est toujours le produit de la synthèse monothétique qui œuvre sous la rubrique de l'attention ${ }^{16}$.

5. L'attention est mobile et labile. Les actes d'attention jouent les uns sur les autres. L'attention peut bien définir les contours d'une expérience - un Ici-Maintenant-Ainsi déterminé -, mais en retour cette définition constitue le point de départ de nouveaux actes d'attention. Le sens défini n'est pas un

13. Ce sera aussi le cas dans la considération du projet lié à l'agir : se projeter, c'est d'abord fantasmer ce qui aura été, c'est produire une récapitulation fictive de ce qui se sera passé. Cf. Aufbau, §9, p. 55 sq.

14. Ibid.

15. Aufbau, $\S 7$, p. 43 sq.

16. Aufbau, § 11, p. 71 sq. 
produit isolé, il est relatif à des contextes de sens (Sinnzusammenhänge) et des contextes d'expérience (Erfahrungszusammenhänge).

Cette phénoménologie de l'attention, commandée par les exigences qui sont celles de l'entreprise de l'Aufbau, à savoir rendre compte du concept sociologique de « sens subjectivement visé », délivre donc l'attention du double paradigme «volontariste » et " intentionnaliste » (au moins dans la mesure où l'attention n'est plus directement pensée par rapport à la perception). Elle ne voit plus dans l'attention un acte particulier du sujet par rapport à tel ou tel objet, mais « cette attitude fondamentale que le moi adopte à l'égard de son flux de vécus au cours du Maintenant et Ainsi du moment ${ }^{17}$ ». On mesure à ce point tout le legs des considérations de Bergson relatives à l'attention à la vie, c'est-à-dire à cette tension fondamentale de l'esprit par rapport à la vie, tension susceptible de degrés, s'extériorisant en action ou $\mathrm{s}^{\prime}$ intériorisant en connaissance ${ }^{18}$. L'attention n'est plus considérée comme une activité consciente qui va de soi et qui serait acceptée telle quelle, comme une donnée psychologique parmi d'autres; elle est reconduite à un fond d'expérience, elle est ressaisie dans le cadre d'une opposition générale entre vie et pensée. L'attention, c'est donc ce qui initie l'orientation de la conscience dans l'expérience.

\section{De la théorie de l'attention à la théorie des systèmes de pertinence}

À partir des années 1940, Schütz développe une théorie des systèmes de pertinence. Celle-ci figure dans un texte inachevé intitulé Reflections on the Problem of Relevance ${ }^{19}$, ainsi que dans l'ultime présentation de l'œuvre qui fut achevée, après sa mort seulement, par son élève Thomas Luckmann sous le titre Die Strukturen der Lebenswelt ${ }^{20}$. On peut considérer que cette théorie des systèmes de pertinence constitue une forme d'approfondissement et de redéploiement de la phénoménologie de l'attention exposée dans l'Aufbau. D'un certain point de vue, tout se passe comme si Schütz avait effectivement donné suite à une remarque formulée à la fin du paragraphe consacré à la

17. Aufbau, §13, p. 77 .

18. Ce concept cardinal de la philosophie de Bergson apparaît dans l'avant-propos de Matière et mémoire, Paris, PUF, 2008, p. 7. Schütz y fait explicitement référence, cf. Aufbau, § 13, p. 77.

19. Les esquisses qui nous sont restées ont été rédigées au cours de l'été 1947: A. Schütz, Reflections on the Problem of Relevance, éd. par R. Zaner, New Haven/London, Yale University Press, 1970 (abrégé Relevance) ; tr. all. par A. von Baeyer, Das Problem der Relevanz, Frankfurt, Suhrkamp, 1971. Sur la théorie des systèmes de pertinence : R. Cox, Schutz's Theory of Relevance, M. Nijhoff, 1978.

20. A. Schütz, T. Luckmann, The Structures of the Life-World, Evanston, Northwestern University Press, 1973 (vol. I) et 1989 (vol. II) pour la traduction anglaise ; Die Strukturen der Lebenswelt, Frankfurt, Suhrkamp, 1974 (vol. I) et 1983 (vol. II) pour l'édition allemande. Nous citons la réédition du texte en un volume chez UVK Verlagsgesellschaft (Konstanz), 2003 (abrégé SL). 


\section{L'attention}

question de l'attention dans l'Aufbau, où il notait qu'il avait découvert le point de départ décisif d'une analyse phénoménologique de l'attention, mais qu'il pouvait se dispenser de poursuivre son investigation compte tenu des impératifs qui étaient les siens ${ }^{21}$.

Mais il convient de noter que le cadre de l'investigation n'est plus vraiment le même que celui-ci de l'Aufbau. En effet, la théorie des systèmes de pertinence relève avant tout du projet d'une description du «monde allant de soi » qu'elle devait inaugurer ${ }^{22}$. Sous cette perspective, Schütz entendait rendre compte de l'institution du monde familier et quotidien, du fonctionnement de l'attitude naturelle qui y préside, de la genèse des réserves d'expérience et de la production d'une connaissance habituelle constamment mobilisées, mais œuvrant de manière quasi inconsciente. Il ne $\mathrm{s}^{\prime}$ agit donc plus de produire une théorie phénoménologique de l'expérience subjective en vue d'une «fondation » philosophie de la sociologie, mais bien de développer une anthropologie philosophique, phénoménologiquement inspirée, décrivant le fonctionnement de l'attitude naturelle. Pour autant, les considérations développées par Schütz ne périment pas les vues adoptées dans l'Aufbau : elles constituent plutôt un prolongement significatif, en ce qu'il met l'accent sur le caractère structuré du rapport de l'attention à l'expérience.

En effet, la question de l'attention est désormais instruite à partir de l'expérience antéprédicative qui la structure. Ce n'est plus seulement l'acte attentionnel qu'il s'agit d'analyser en déterminant sa nature, sa portée et ses modalités, mais plus largement le rôle joué par l'attention dans l'institution du monde «allant de soi ». Dès lors, l'acte attentionnel n'est plus envisagé pour lui-même : il se détache sur le fond d'une vie intentionnelle largement passive, inconsciente et incorporée, constituée d'expériences inaperçues (seen but unnoticed) et tenues pour allant de soi (taken for granted). L'attention, acte sélectif et distinctif, présuppose l'existence d'un arrière-plan cognitif non thématisé. C'est par le biais de l'ensemble de ce à quoi nous n'accordons pas d'importance que l'attention doit être abordée. Certes, la question initiale de l'attention - celle de l'accès au phénoménal - demeure fondamentale, mais elle doit être reprise à partir de la considération de ce qui ne fait pas question à nos yeux. C'est en somme toute la problématique phénoménologique qui se trouve renouvelée en son cœur, c'est-à-dire au niveau même de ce que l'on tient pour "phénomène", pour apparaissant ou non à la conscience. Il ne s'agit plus de penser l'émergence de la phénoménalité par le biais d'une attention qui s'achève comme thématisation, saisie de l'objet dans son unité,

21. «Nous ne pourrons indiquer que le point de départ d'une analyse phénoménologique de l'attention dont la conduite in extenso excède le cadre de cette recherche. Il nous suffit d'avoir gagné, à travers la considération des modifications attentionnelles auxquelles les orientations du regard vers les vécus passés sont soumises, un point de départ pour une théorie de la constitution du sens spécifique de ces vécus toujours particuliers. " Aufbau, § 13, p. 78.

22. Le titre complet de l'ouvrage projeté aurait été le suivant: The World as Taken for Granted: Toward a Phenomenology of the Natural Attitude. 
mais bien plutôt le rapport entre la thématisation et ce qui gît à son horizon ou à son arrière-plan.

En effet, dans l'attitude naturelle, nous présupposons constamment l'existence d'un monde de la vie familier, quotidien, dont l'évidence n'est pas remise en cause. L'homme qui se tient dans l'attitude naturelle accorde au monde le bénéfice de la Selbstverständlichkeit, une auto-compréhensibilité qui aveugle sur ce qu'il est véritablement. À ses yeux, le monde de la vie est ce qui va de soi, ce qu'il laisse pour ininterrogé, alors même qu'il constitue la toile de fond de l'ensemble de mes activités. Certes, il arrive parfois que nous rencontrions, dans l'attitude naturelle, des échecs, des déceptions qui sont autant de heurts avec la réalité primordiale, de résistances que $\mathrm{m}^{\prime}$ oppose le monde de la vie quotidienne. Mais les problèmes pratiques que nous rencontrons au quotidien, s'ils brisent parfois la "chaîne d'évidence " (Selbstverständlichkeitskette) qui s'y développe en permanence, ne font pas du monde de la vie une réalité problématique en elle-même ${ }^{23}$.

Ce que Schütz analyse sous la rubrique des «structures de pertinence » concerne la dimension proprement subjective de cette institution cognitive d'une réalité non problématique. Le terme de "pertinence » désigne ici un ensemble de potentialités attentionnelles susceptibles de satisfaire aux exigences objectives et subjectives d'une situation. L'ensemble de notre expérience et de nos actions sont ainsi réglées, projetées par avance, définies par des structures de pertinence qui les rendent non problématiques. Les structures de pertinence définissent le cadre cognitif d'un ajustement au monde qui doit toujours être gagné, faire l'objet de confirmations nouvelles, mais qui peut aussi légitimement miser sur les réserves d'expériences acquises, sur une évaluation raisonnable du vraisemblable, du probable et du convenable.

Pour Schütz, l'arrière-plan cognitif sur le fond duquel s'enlèvent nos actes d'attention structure, oriente et détermine notre définition et notre maîtrise des situations ${ }^{24}$. Le sujet de l'attention n'est plus seulement, comme chez Husserl, l'ego transcendantal en tant que foyer de la vie intentionnelle,

23. A. Schütz, T. Luckmann, SL, p. 39.

24. Cf. W.I. Thomas, The Unadjusted Girl with cases and standpoint for behavior analysis, Boston, Little Brown and Company, 1923, où Thomas, l'une des plus éminentes figures de l'École de Chicago, précise son «théorème », selon lequel «quand des individus définissent une situation comme réelle, elle est réelle dans ses conséquences ». La définition de la situation est en fait un «examen" et une «délibération » qui précède "l'auto-détermination » du comportement. Il importe assez peu que l'interprétation de la situation soit correcte ou non : ce qui prime ici, c'est l'inscription pratique qui est rendue possible par la définition de la situation. Définir la situation, ce n'est donc pas seulement délimiter un ensemble unitaire de circonstances dont la prise en compte importe pour la perception de la situation et pour l'action engagée. Définir la situation, c'est bien plutôt saisir le sens de ce qui se passe et, plus positivement, identifier des possibles ouverts dans des circonstances données. La définition de la situation, ainsi caractérisée comme produit d'un processus individuel et volontaire, est toujours tendue entre la «définition spontanée que les membres de la société organisée » donnent de la situation et « celle que la société pourvoit ». Entre les deux, ce qui se joue, c'est la personnalité de l'individu, relative à une série de définitions de situation. 


\section{L'attention}

mais l'individu pris dans un parcours biographique, dans une succession de situation à maîtriser et dans la succession corrélative des expériences qui se sédimentent en lui. Schütz est cependant fidèle à cet acquis de la phénoménologie génétique de Husserl, selon lequel il y a toujours quelque chose qui reste de l'acte accompli et se sédimente dans l'expérience passive $\mathrm{du}$ sujet. Toute thématisation réussie implique ainsi des systèmes de typification et de pertinence qui seront réactivés au contact des événements et des situations, dans des conditions analogues. Les systèmes de pertinence sont régis par un principe de répétabilité implicite : ce qui a réussi une fois peut être repris, répété sous conditions équivalentes. Les systèmes de pertinence définissent des dispositions pratiques et théoriques, des anticipations qui conditionnent en retour l'exercice de nouvelles thématisations. Les structures de pertinence ne sont pas statiques, définitives et raidement déterministes: elles procèdent $d^{\prime}$ une genèse biographique particulière relative à une multitude de problèmes théoriques et pratiques rencontrés par l'individu ; elles sont également l'objet de remaniements permanents.

C'est à ce point qu'il convient de souligner l'importance d'une seconde réorientation du propos. En s'efforçant de pratiquer une phénoménologie redéfinie comme analyse réflexive de l'attitude naturelle, Schütz ne s'est pas contenté de donner une portée nouvelle à la théorie du monde de la vie. Il reconnaît et assume le bien-fondé de conceptions pragmatistes qui, dans le jeu de leurs différences et de leur complémentarité, constituent ici un précieux renfort (on songe à James, Bergson et Scheler dans un premier temps, puis, après l'exil aux États-Unis, aux sociologues et anthropologues américains C.H. Cooley, G.H. Mead et J. Dewey, entre autres ${ }^{25}$ ). Pour Schütz, la « réalité primordiale » sur quoi s'appuie l'attitude naturelle est plus largement $\mathrm{d}^{\prime}$ ordre pratique et social. Le sujet qui se rapporte au monde de la vie est désormais avant tout un agent, un soi travaillant, un working self directement en prise avec le monde, par le biais de performances et de mouvements corporels qui produisent des effets et des résultats escomptés. Pour Schütz, notre rapport au monde de la vie est, d'emblée, foncièrement pratique. Le monde de la vie est ainsi ce monde où à proprement parler se joue ma vie. Notre existence ne consiste pas à endurer le monde passivement. Bien au contraire : le monde de la vie renvoie à une expérience vitale du monde, à ce que j'entreprends pour assurer ma subsistance et ma survie. Le monde de la vie est la réalité avec laquelle je dois composer, le cadre nécessaire de réalisation de mes actions, entre réussites et échecs. Le monde de la vie est ainsi d'un côté, pratiquement défini par mes projets, mes décisions, mes

25. Sur ce que l'on a pu appeler le « tournant pragmatiste », cf. : D. Cefaï, op. cit., p. 75. Ce tournant de l'œuvre s'opère notamment dans l'article de 1943 intitulé "Sur les réalités multiples» (A. Schütz, "On Multiple Realities », CP I, p. 113-135 [ "Sur les réalités multiples », CQ, p. 103167]) que complète utilement celui de 1954 intitulé «Symbole, réalité et société » (A. Schütz, "Symbol, Reality, and Society », CP I, p. 113-135). Cette orientation pragmatiste sera accentuée dans les derniers textes de Schütz. 
actions effectives ou potentielles; de l'autre, il est aussi ce qui me résiste, ce qui fait obstacle, jusqu'à l'échec de l'action entreprise.

Schütz rompt ainsi, plus nettement encore que dans l'Aufbau, avec le paradigme perceptif qui sous-tendait la théorie husserlienne de l'attention. Une structure de pertinence constitue une forme de solution déjà éprouvée devant tel ou tel problème. Elle ne répond donc pas prioritairement à un besoin théorique, mais bien plutôt à des exigences pratiques. Pour l'homme de l'attitude naturelle qui est toujours tenu de répondre immédiatement à la situation, l'essentiel est en effet de choisir opportunément au sein de ce qui se donne à eux, en instituant des formes de partage de l'expérience : entre l'essentiel et l'insignifiant, le familier et l'étranger, le certain et l'incertain, le convenu et l'incongru, etc. Les structures de pertinence, sur le fondement des réserves d'expérience, garantissent une sorte de mise en forme préalable de l'expérience et de nos possibilités d'action.

Ce sont ces deux données - la réorientation du propos en direction d'une anthropologie philosophique de la vie quotidienne ; l'assomption de l'héritage du pragmatisme - qui justifient le changement apparent de thématique et la transition opérée d'une théorie de l'attention vers une théorie de la pertinence. Il ne s'agit plus seulement de décrire le fonctionnement de l'activité de thématisation, mais bien de comprendre comment quelque chose prend de l'importance à nos yeux par rapport à d'autres qui n'en ont plus. Sous la rubrique de la pertinence, c'est donc le rapport spécifique qui se joue, dans l'ordre des synthèses passives ou de l'expérience antéprédicative, entre le thème et son horizon qui doit être étudié.

Sur ces fondements, l'analyse structurale des différents types de pertinence qui régissent le travail de l'attention - mais aussi la constitution du sens de l'expérience - établit une distinction entre la pertinence motivationnelle, la pertinence topique ou thématique, et la pertinence interprétative.

1. La «pertinence motivationnelle» (motivational relevancy) est la plus générale des structures de pertinence. Elle sous-tend les structures de pertinence thématique et interprétative.

Elle a pour caractéristique d'être relative aux intérêts pragmatiques qui déterminent la visée intentionnelle de l'action dans une situation donnée. Le faire, effectif ou projeté, est la « raison » véritable du savoir mobilisé. La pertinence motivationnelle est ainsi à l'œuvre dès que le sujet s'efforce de résoudre un quelconque problème d'ordre pratique ou théorique, ou encore tant que $l^{\prime}$ « intérêt » porté à une situation n'a pas trouvé entière satisfaction ${ }^{26}$. L'ensemble de notre activité de connaissance et de notre rapport au monde et ceci vaut également pour l'exercice de l'attention - s'ordonne à un sens pratique qui met à l'épreuve la validité des opérations de compréhension et

26. A. Schütz, SL, p. 286 et Relevance, p. 64-65. La notion d'intérêt doit être comprise en un sens non utilitariste. Elle est empruntée à E. Husserl, Expérience et jugement, Paris, PUF, 1991, § 17 et 20. Elle renvoie à la fois à une dimension de prédonation passive de l'objet et une activité de thématisation de l'objet qui peut être volontaire ou non, mais qui aboutit à un sentiment de satisfaction ou à la position de buts. 


\section{L'attention}

d'explication et qui recherche l'efficacité de l'action entreprise. Les structures de pertinence permettent l'orientation pragmatique de l'individu et répondent ainsi aux exigences de la Wirkwelt, ce "monde du travail », dont la réalité pratique est constituée par les interactions et les interlocutions des différents acteurs ${ }^{27}$.

Les structures de pertinence motivationnelle ont ainsi pour fonction de s'exprimer à travers la désignation de motifs à cause de (Weilmotive) et de motifs en vue de (Umzumotive). Elles mobilisent des schèmes motivationnels qui réfèrent l'action à son origine ou qui la projette dans l'avenir. La définition des situations, la constitution des contextes de sens, l'engagement de l'action s'ouvrent sur un double horizon causal et téléologique. Ils s'inscrivent ainsi dans la continuité du parcours biographique de l'individu, dans une série de «chaînes de motivation » (Motivationskette ${ }^{28}$ ). Les structures de pertinence motivationnelle alimentent ainsi une vie pratique qui s'oriente immédiatement selon les situations rencontrées, en fonction des contextes de sens établis et en vue de ce qui peut ou doit être fait.

Concrètement, les structures de pertinence opèrent un travail de sélection qui identifie, dans la structure du monde donné d'avance et au sein des réserves d'expérience du sujet, un ensemble d'éléments qui seront les plus à même de constituer une réponse appropriée à la situation pratique rencontrée. La pertinence motivationnelle ordonne les réserves d'expérience actuelles et effectives en fonction de la structure situationnelle du monde. En effet, selon la situation, le système de pertinence pourra privilégier la spontanéité du sujet (dans le cas d'une situation qui procède de notre propre choix) ou la réceptivité (dans le cas de ces situations «imposées » que sont le deuil, la maladie, les catastrophes naturelles, etc ${ }^{29}$.). Les éléments retenus dans ces réserves d'expérience peuvent être tirés d'une « connaissance sur » (knowledge about), c'est-à-dire d'une connaissance experte, informée et précise de la réalité. Mais le plus souvent, au-delà du domaine limité des savoirs maîtrisés, nous mobiliserons des savoirs vagues, des " connaissances de » (knowledge by acquaintance), des informations superficielles et relatives qui nous permettent de nous débrouiller ${ }^{30}$. À la limite, nous aurons recours à de simples croyances. Aussi longtemps que ces différentes composantes des réserves d'expérience suffisent, l'individu peut agir d'une manière qui n'est pas en elle-même problématique.

2. Sitôt qu'un nouveau problème survient, les structures de pertinence topique ou thématique entrent en jeu. Il ne s'agit alors plus de préserver le

27. A. Schütz, « On Multiple Realities », CP I, p. 218 [« Sur les réalités multiples », CQ, p. 116].

28. A. Schütz, SL, p. 291.

29. Schütz établit ainsi une distinction entre l'intrinsic relevance (pertinence intrinsèque) et l'imposed relevance (pertinence imposée) : A. Schütz, «The Well-Informed Citizen », CP III, p. 126-127 ; tr. T. Blin, Phénoménologie et sociologie compréhensive. Sur Alfred Schütz, Paris, L'Harmattan, 1995, p. $120-121$.

30. Cette distinction est empruntée à W. James, The Principles of Psychology, vol. I, New York,H. Holt and Co., 1904, p. 459 ; tr. fr. Précis de psychologie, M. Rivière, 1909, p. 220-221. 
caractère non problématique de l'action et de la pensée quotidienne, mais bien de faire droit à ce qui fait désormais question. Ces structures de pertinence apportent une réponse cognitive aux perturbations rencontrées dans le monde social, aux formes inédites d'expérience. L'analyse de ces structures de pertinence est particulièrement importante pour notre propos, puisque ce sont elles qui commandent la mise en relief du thème de l'attention.

Dans le cas de la «pertinence imposée », notre intérêt se réoriente à la faveur d'un événement. Schütz distingue ainsi quatre formes fondamentales de changement de pertinence imposé : à l'occasion de l'apparition d'une étrangeté qui tranche avec une réalité familière (1); dans la découverte de nouveaux thèmes en "sautant " d'un domaine de réalité à un autre (2) ou encore via les modifications de la «tension de conscience » (3); à la faveur d'une réorientation socialement contrainte de l'intérêt $(4)^{31}$.

Schütz distingue de ce cas celui de la «pertinence motivée », liée à un déplacement volontaire de l'attention. Un changement de pertinence motivé se produit lorsque nous dirigeons volontairement notre attention au cours d'un examen méthodique (par exemple, lorsque nous examinons attentivement un tableau dans tous ses détails). La conversion attentionnelle (Zuwendung) engendre alors des modifications qui vont entraîner une réorganisation de la structure thème-contexte. Ces réorganisations peuvent être de différentes natures : approfondissement d'un thème ; superposition d'un nouveau thème, abandon du thème et refocalisation. Ces réaménagements se produisent à l'horizon externe de la thématisation (contiguïté spatiale ou continuité temporelle) ou au sein de son horizon interne (dans le cas d'une description attentive ${ }^{32}$.

3. Enfin, la pertinence interprétative est celle qui nous permet de mettre en œuvre des schèmes interprétatifs qui vont décider du sens que prend pour nous telle expérience. Il n'est alors plus seulement question de la saisie d'une unité objective, mais plus largement de la modalité particulière du rapport établi entre le sujet et l'objet thématisé.

Autour de la saisie thématique d'un objet défini, d'un thème précis (un tableau par exemple) se déploie tout un champ pratico-sensible (le tableau dans le musée), ainsi que des anticipations de sens (son appartenance possible à tel ou tel courant, etc.). La pertinence interprétative ne détermine pas la focalisation de l'attention, mais l'application des schèmes interprétatifs sélectionnés en fonction des particularités de la situation.

En ce sens, la pertinence interprétative est relative à notre parcours biographique en ce qu'elle définit notre manière personnelle de nous rapporter à tel objet ou à tel événement. Mais cette pertinence interprétative est aussi socialement constituée. La genèse de ce type de structure de pertinence n'est pas strictement personnelle ou purement privée : elles sont acquises par le biais d'un apprentissage des pratiques, des savoir-faire, des

31. A. Schütz, $S L$, p. $258-262$

32. A. Schütz, $S L$, p. 263-269. 


\section{L'attention}

usages. Elles renvoient à un sens commun qui norme l'exercice de l'attention et au-delà, la constitution du sens de l'expérience. Selon Schütz, il existe au fondement de toute connaissance se rapportant au monde social une connaissance commune: on ne peut pas agir et penser sans mobiliser une réserve d'expériences préalables qui se présentent sous forme de «connaissances disponibles » et fonctionnent comme "schèmes de référence ", au sein d'un stock de connaissances disponibles (Wissensvorrat ou stock of knowledge).

Ce sens commun constitutif du monde social, partagé à divers degrés par les différents acteurs, se caractérise par sa typicalité. Schütz étend aux acteurs du monde social les remarques de Weber sur la construction idéaltypique: les acteurs se livrent sans cesse à une activité de typification du monde social, au cours de laquelle les objets du monde social sont repérés et ordonnés selon leur traits constitutifs généraux. Leur fonction est essentiellement pratique : elles structurent pratiquement le monde social en attentes raisonnables. Mais si le sens commun structure ainsi le monde social en attentes raisonnables, les typifications, maximes et définitions n'ont pourtant pas de déterminations définitives et sont potentiellement équivoques : ce qui décide de leur application, c'est le contexte, via l'application des schèmes interprétatifs pertinents. La théorie de la pertinence interprétative déborde ici clairement le cadre de la théorie de l'attention pour déployer une analyse structurale et génétique du sens commun constitutif de la réalité sociale ${ }^{33}$.

En un sens et d'un point de vue strictement phénoménologique, Schütz tire donc toutes les conséquences du repérage husserlien du rôle de la sphère antéprédicative, qui invite à penser l'acte de l'attention à partir de l'expérience sédimentée du sujet et sur le fond de structures cognitives instituées et en réaménagement constant. On voit quelle est la distance ainsi établie par rapport aux théories classiques de l'attention. L'attention n'est pas le produit du libre-arbitre créateur du sujet, elle s'exerce sous conditions, en fonction de structures de pertinence qui lui préexistent et qui sont régies par la situation biographique du sujet, c'est-à-dire par son histoire sédimentée et son insertion au sein d'une situation, laquelle n'est jamais $\mathrm{qu}^{\prime}$ une somme de possibles pratiques. Schütz pose ainsi les bases d'une conception de l'attention qui fait sans doute mieux droit à ses dimensions pratico-sensible et sociale.

33. A. Schütz, SL, p. 272-285. 